\title{
Application of Artificial Intelligence for Rapid Prevention of Epidemic Diseases (COVID-19)
}

Faridoddin Shariaty ( $\sim$ Shariaty3@gmail.com )

Peter the Great Saint-Petersburg Polytechnic University https://orcid.org/0000-0002-7060-8826

\section{Shamsoddin Shariaty}

Shahid Beheshti University

\section{Samaneh Navvabi}

Saint-petersburg state University https://orcid.org/0000-0001-8825-5793

\section{Mojtaba Najafi Oshnari}

Saint-petersburg state University https://orcid.org/0000-0003-3949-6608

\section{Boris Novikov}

Saint-Petersburg HSE University https://orcid.org/0000-0003-4657-0757

\section{Systematic Review}

Keywords: Diagnostic imaging, COVID-19, respiratory infection, Computer Aided Detection system (CADs), radiology, CT images, review

Posted Date: February 1st, 2022

DOI: https://doi.org/10.21203/rs.3.rs-1309509/v1

License: (c) (i) This work is licensed under a Creative Commons Attribution 4.0 International License.

Read Full License 


\title{
1. Application of Artificial Intelligence for Rapid Prevention of Epidemic Diseases (COVID-19)
}

\author{
3 Faridoddin Shariaty ${ }^{* \dagger}$, Shamsoddin Shariaty ${ }^{2 \dagger}$, Samaneh Navvabi ${ }^{3}$, Mojtaba Najafi \\ Oshnari $^{3}$, and Boris Novikov ${ }^{4}$ \\ ${ }^{1}$ Peter the Great Saint-Petersburg Polytechnic University, Saint-Petersburg, Russia. \\ ${ }^{2}$ Shahid Beheshti University, Tehran, Iran. \\ ${ }^{3}$ Saint-petersburg state University, Saint-Petersburg, Russia. \\ ${ }^{4}$ Saint-Petersburg HSE University, Saint-Petersburg, Russia.
}

\begin{abstract}
Background: Epidemic diseases are hazardous in terms of a rapid outbreak. Rapid control of these diseases by finding patients and quarantine and treatment can be the only tool to reduce the number of cases and mortality at the beginning of the outbreak, in the absence of therapy and vaccines. COVID-19 (coronavirus) is a deadly viral disease that causes severe respiratory illness and spreads through the air. Artificial intelligence (AI) technologies have played an essential role in solving complex problems. The use of these technologies in response to the challenges posed by the COVID-19 epidemic can reduce the effects of epidemics in various contexts.

Objective: The purpose of this article is to review the applications of artificial intelligence in cases of contagious disease. In this work, COVID-19 disease has been used as an example of dangerous infectious diseases (while the studied methods can be used for all contagious diseases), and a systematic review of the literature on the role of artificial intelligence as COVID-19 has become a comprehensive and critical technology for combating epidemiology, diagnosis, and disease progression.

Methods: A complete search of the literature has been done using the databases of PubMed, Scopus, Web of Science, and Google Scholar, and other sources. In this work, the aim is to review articles that the authors believe can be helpful in the prevention of infectious diseases in the event of an outbreak of artificial intelligence in the prevention of more casualties. The first steps needed in a flurry of a disease (including coronavirus) include identifying the primary sufferers and isolating them from the public and examining the illness and how the disease has progressed. In these stages, artificial intelligence can very effectively help the medical community and even the government prevent an epidemic. In this study, the keywords COVID-19 and artificial intelligence and infectious diseases have been used.

Results: During our literature search, we came across 73 papers. Researchers analyzed studies examining the diagnostic roles and imaging features of patients with COVID-19. The
\end{abstract}


latter were scanned using CT or ultrasound scans, chest radiographs, or positron emission tomography/computed tomography (PET/CT) scans. Chest x-ray and CT scan are the imaging modalities that are most widely utilized for the diagnosis and management of COVID-19 patients, with chest CT scan being more accurate and sensitive in diagnosing COVID-19 at an early stage. Only a handful of studies have looked into the roles of ultrasonography and PET/CT scans in diagnosing COVID-19 infection.

Conclusions: We gathered research from the existing COVID-19 literature that employed artificial intelligence-based methodologies to give insights into various domains of COVID-19 in this systematic review. Our findings indicate critical variables, data formats, and COVID-19 sources to help with clinical research and translation. Findings from this study may also assist in reducing the harm caused by the pandemic in the case of such epidemic diseases in the future.

Keyword: Diagnostic imaging, COVID-19, respiratory infection, Computer Aided Detection system (CADs), radiology, CT images, review.

\section{Introduction}

COVID-19 is a global health crisis, and according to the World Health Organization, as of October 15, 2021, approximately 16 million people were infected, and more than 666,000 deaths were reported worldwide (1). High degrees of variance has been reported in symptoms of COVID-19, from mild flu to acute respiratory distress syndrome (ARDS) or severe pneumonia $(2 ; 3 ; 4)$. Effective drugs and vaccinations are required immediately to treat and prevent COVID-19. Due to the lack of valid therapeutic drugs, most inhibitory methods used to prevent disease transmission rely on social isolation, quarantine procedures, and lockdown policies (5; 6). COVID-19 transmission has slowed but not ceased as a result. Additionally, with the ease restrictions, the concerns about further waves of infection rise $(7 ; 8)$. To prevent the onset of subsequent COVID-19 waves, advanced control measures such as contact tracking and point detection are needed $(9 ; 10)$.

Artificial intelligence (AI) refers to various technologies that try to simulate human cognitive capabilities and intelligent behaviors. Machine learning (ML) is an artificial intelligence discipline that focuses on techniques that allow computers to construct patterns for complicated connections or observed data patterns without explicit preparation. Deep Learning (DL), a subset of ML, motivates biological neural networks to handle a broad range of complicated problems, including medical imaging and natural language processing (NLP) categorization, with greater power and flexibility than models that achieve regular ML (11).

The medical sector is seeking innovative methods to detect and manage the spread of COVID-19 during this worldwide health crisis (Coronavirus). Artificial intelligence is one of the technologies that can quickly track viral propagation, identify at-risk patients, and be beneficial in real-time infection management. By examining past patient data, it may also forecast death. Through population screening, medical assistance, information, and infection control recommendations, artificial intelligence can contribute in the dealing with the virus $(12 ; 13 ; 14)$. As an evidence-based medical tool, this technology can improve the planning, treatment, and reported results of COVID-19 patients. 
This paper concentrates on the emerging COVID-19 pandemic and how to overcome issues during an outbreak using contemporary AI and ML technology. We present a thorough review of research on the model and technologies utilized to address the emerging COVID-19 outbreak. These studies further explore the sorts of AI and ML methods that have recently employed integration and the types of data sets, the ultimate performance of each suggested model, and the merits and downsides of new methodologies.

\section{Methods}

PRISMA (Preferred Reporting Items for Systematic Reviews and Meta-Analyses) principles were used to prepare and report this systematic literature review (15).

\subsection{Eligibility Criteria}

The study concentrated on peer-reviewed papers and pre-publication that employed artificial intelligence approaches to investigate and address the COVID-19 problem at several scales, such as diagnostic, prognosis, and disease prognosis.

\subsection{Data Sources and Search Strategy}

The databases PubMed, Web of Science, and CINAHL were searched. The search was restricted to research publications published in English and valid or pre-published journals or conference papers from December 1, 2019, to June 27, 2020. The syntax was developed with the assistance of a professional librarian and includes the following search terms: "CORONAVIRUS", "COVID-19", "covid19", "cov-19", "cov19", "Acute Coronavirus Acute Respiratory Syndrome" 2 "," Coronavirus in Wuhan "," Wuhan Seafood Market Pneumonia Virus ", Coronavirus Virus 2019 "," SARS-CoV-2 ", SARS2 ", SARS-2 "," 2019-nCoV".

\subsection{Study Selection}

Following the systematic search, 285 publications were found. 60 duplicates were deleted, leaving 225 possibly relevant papers for the title and abstract. After reviewing these papers, 102 further publications were deleted, leaving 123 publications for full-text examination. These papers were then reviewed for eligibility, and a total of 73 were included in the final analysis. The databases PubMed, Web of Science, and CINAHL were searched. The search was restricted to research publications published in English and valid or pre-published journals or conference papers from December 1, 2019, to Oct 27, 2021. The syntax was developed with the assistance of a professional librarian and includes the following search terms: "CORONAVIRUS", "COVID-19", "Acute Coronavirus Acute Respiratory Syndrome", Coronavirus in Wuhan", "Coronavirus Virus 2019", "SARS-CoV2", "epidemic", "pandemic". 


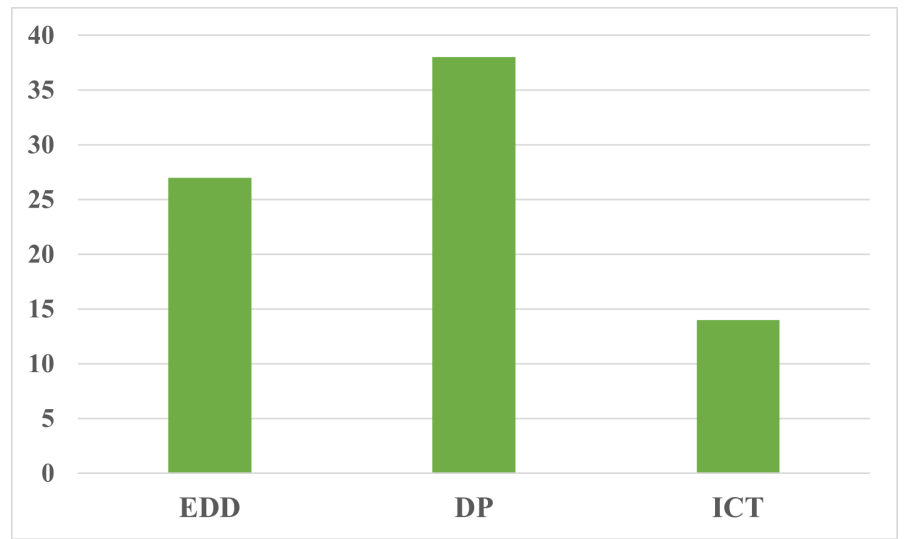

Figure 1: Caption

\subsection{Data Collection and Analyses}

The included research that employed artificial intelligence strategies to address the COVID-19 outbreak was subjected to quantitative and qualitative descriptive analysis. The research was divided into three categories based on their application: (1) early detection and diagnosis (EDD), (2) disease progression (DP), and (3) Individual contact tracing (ICT). Research on EDD was subjected to qualitative analysis, whereas studies on DP and ICT were subjected to descriptive quantitative analysis.

The search strategy led to the discovery of 285 articles, of which 73 articles were selected for further analysis. These papers were divided into three categories based on the applicability of artificial intelligence to the COVID-19 crisis: early detection and diagnosis, disease progression, and individual contact tracing. Of the 73 research studies, 27 (37\%) focused on detecting COVID-19 in medical imaging to differentiate Covid 19 from other lung diseases, categorized as early detection and diagnosis. Then, in 32 of the 73 cases (44\%), artificial intelligence approaches were utilized to estimate the progression of COVID-19 using radiological images of patients or laboratory data. Finally, 14 (19 percents) of the 73 research focusing on tracing patients with COVID-19 and presenting virus-infected locations and suspected persons were categorized (Fig. 1).

\section{Application of AI for Rapid Prevention of Epidemic Dis- eases}

\subsection{Early detection and diagnosis (EDD)}

Artificial intelligence can identify unusual symptoms rapidly and inform patients and health authorities.(16;

$17 ; 18)$. This speeds up decision-making and saves money. Applying relevant algorithms contributes to the development of a novel detection and management system for COVID 19 situations. With medical imaging technologies such as computed tomography (CT) and magnetic resonance imaging (MRI) of human body parts, artificial intelligence may be used to diagnose infected patients. In two 


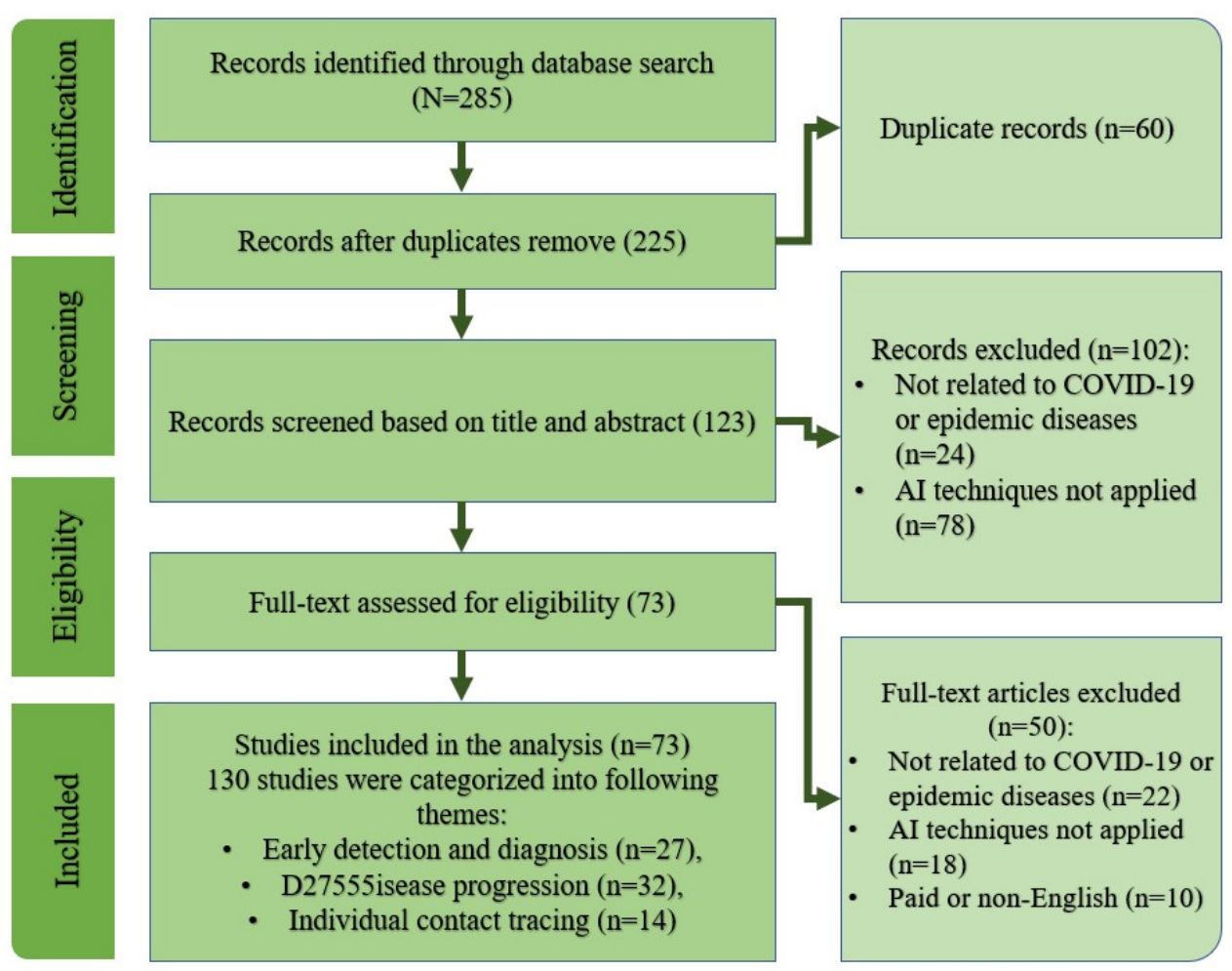

Figure 2: Dispersion of articles reviewed in this work.

parts, we will look into COVID-19 illness and ways for recognizing the virus:

\subsubsection{COVID-19 Detection from Normal cases}

Due to the development of computer systems, these systems can be used in medicine and the diagnosis of disease (19). In recent years, many advances have been made in computer systems and have been widely used to diagnose lung cancer (20). Recently, due to the prevalence of COVID-19 disease (18), these systems can be used for automatic and early diagnosis of this disease. Many systems have recently been designed for this purpose, most of which use machine learning.

Dynamic lung CT of COVID-19 described and summarized in 4 steps (21). In summary, the first four days after early symptoms, the early stage is considered, and GGO can be seen subpleural in the lower lobes unilaterally or bilaterally. Progressing stage is 5-8 days when it is possible to find diffuse GGO spreading over bilateral polylobed. At the peak stage (9-13 days), dense consolidation becomes more common. Finally, when the infection is controlled, absorption occurs (usually after 14 days), consolidation is gradually absorbed, and only GGO remains. These x-ray patterns are essential evidence for CT-based classification and COVID-19 severity assessment.

Several studies aimed at classifying patients with COVID-19 from patients without COVID-19 (including patients with advanced pneumonia and cases without pneumonia). Chen et al. (22) used lung CT scans of 51 COVID-19 patients and 55 patients with other diseases for training a UNet++-based segmentation model, which as a result segments lesions associated with COVID-19. 
The results of this method were: accuracy $=95.2 \%$, sensitivity $=100 \%$, and specificity $=93.6 \%$. In another dataset of 16 patients with viral pneumonia and 11 patients without pneumonia, this model identified all patients with and nine patients without pneumonia. As a result, the time of reading for radiologists was reduced by $65 \%$ using AI results.

A model based on U-Net $+3 \mathrm{D}$ CNN (DeCoVNet) proposed by C. Zheng et al. (23). In this method, lungs are segmented using U-Net, and the result of segmentation is used as 3D CNN input to predict the likelihood of COVID-19. Five hundred forty lung CT scans (i.e., 313 COVID-19 patients and 229 healthy) were used as data for deep learning. This model achieved a sensitivity of $90.7 \%$, a specificity of $91.1 \%$, and an AUC of 0.959 .

Gene et al. (24) used chest computed tomography of 496 cases of COVID-19 and 1385 cases without COVID-19. To segment the lungs and then to cut out positive cases of COVID-19, a two-dimensional CNN-based model is proposed. According to the results, this model achieves a specificity of $95.5 \%$, a sensitivity of $94.1 \%$, and an AUC of 0.979 .

Deep Neural Networks (DNNs) have also been proposed as an approximation approach. This approach is a key option for estimating the solution of a Partial Differential Equation and has been employed for COVID-19 detection using CT scans and chest X-rays (25).

Deep learning-based feature extraction frameworks for automated COVID-19 categorization were compared by Sara H. K., et al. (26). MobileNet, DenseNet, Xception, ResNet, InceptionV3, InceptionResNetV2, VGGNet, and NASNet were selected from a pool of deep convolutional neural networks in order to produce the most accurate feature, which is a crucial component of learning. DenseNet121 feature extractor and Bagging tree classifier were found to be the most accurate, with $99 \%$ classification accuracy. Using a ResNet50 feature extractor trained by LightGBM, the second-best learner had an accuracy of $98 \%$.

\subsubsection{COVID-19 Detection from Similar Diseases}

One of the significant challenges that have garnered considerable attention is the difference between the lung injuries caused by COVID-19, pulmonary edema, and other cases in CT images. It was observed from early descriptions of respiratory failure due to COVID-19 that some patients experienced hypoxemia that was disproportionate to the reported dyspnea or level of radiological opacity, with greater than typical respiratory system compliance and less work of breathing. One idea that has attracted much attention, especially on social media and in medicine, is the notion that lung injury due to COVID-19 is more like pulmonary edema. This conclusion, expanded on social media, has led to further speculation that therapies commonly used to prevent and treat pulmonary edema and other acute altitude sicknesses may benefit patients with lung injuries due to COVID-19. However, a review of the pathophysiology of pulmonary edema and a close examination of the mechanisms of action of the drugs used to treat pulmonary edema should make it clear that the COVID-19 lung injury is not comparable to pulmonary edema and that treatments used for pulmonary edema have no benefit or, worse, cause harm to the patient with COVID-19 (27).

However, pathological studies in pulmonary edema and studies of effective mechanisms and drugs for managing the pulmonary edema disease are not effective in patients with COVID-19 and, in 
some cases, are even harmful, leading to injury to the patient. Given this, it can be concluded that despite the similarities between pulmonary edema and COVID-19 in clinical characteristics such as hypoxemia, radiography opacities and modified lung compliance, the pathophysiological mechanisms of pulmonary edema and COVID-19 in the lungs are essentially different, and the diseases cannot be viewed as equivalent. As a result, while systemically administered pulmonary vasodilators and acetazolamide are beneficial in the treatment of pulmonary edema and acute mountain sickness, they should not be used to treat COVID-19 due to the risk of several adverse effects such as deteriorated ventilation and perfusion adaptation, impaired carbon dioxide transport, systemic hypotension, and increased work of breathing (28).

Thousands of images per patient are generated in current clinical practices, making it cumbersome for doctors to analyze all the data $(19 ; 20)$. In addition, human interpretation of medical images can produce errors so that not all information in the image is recognized. The advances made in computer systems allow drawing on the expertise of radiologists to extract data from medical images $(29 ; 30)$. Given the rapid spread of COVID-19, using Machine Learning (ML) algorithms for processing chest CT scans might help to identify the defining clinical characteristics and severity of the disease (31). Although CT provides rich pathological information, only a qualitative assessment was made in the radiological reports, as there are no computer-aided tools for quantifying the infection regions and their longitudinal changes (32). Developing computer vision systems aids in medical applications such as image quality enhancement, organ segmentation, and organ texture classification.(21; 33). Many papers have been written in recent years (2019-2021) about the automatic detection of COVID-19 using CT scan images and machine learning algorithms to distinguish patients with COVID-19 from non-infected patients (34).

Due to the radiological similarity of COVID-19 to common pneumonia and viral pneumonia, differentiation in facilitating the screening process would be more useful in clinical practice. Thus, Wang et al. (35) proposed a CNN model for the classification of these two diseases using 99 lung CT scans in which exist 44 COVID-19 patients and 55 typical viral pneumonia). The test dataset shows an overall accuracy, specificity, and sensitivity of $73.1 \%, 67.0 \%$, and $74.0 \%$.

Ying et al. (36) proposed deep learning computed tomography system (called Deep Pneumonia using ResNet50) for identifying COVID-19 patients in patients with bacterial pneumonia and healthy people. Chest CT data from 88 COVID-19 patients, 101 bacterial pneumonia patients, and 86 healthy people are used as data for learning the network. In addition, pieces of full lungs from CT images are obtained of the chest as input data of deep learning. The model achieved good results with $86.0 \%$ accuracy for classifying patients with COVID-19 or patients with bacterial pneumonia and $94.0 \%$ accuracy for diagnosing pneumonia (COVID-19 or healthy).

$\mathrm{Xu}$ et al. (37) use lung CT scans of 219 patients with COVID-19, 224, and 175 influenzas A and healthy cases. A V-Net-based deep learning model at first used for the segmentation of infected areas. Infected area patches were then sent to the Resnet-18 network and indications of the relative infection distance from the edge, and as output, they had three groups. The overall accuracy of the model was $86.7 \%$.

Shi et al. (38) used a chest CT scan of 2685 patients, consisting of 1658 patients with COVID-19 and 1027 patients with generalized pneumonia. At the pre-processing step, VB-Net (39) used for 
segmentation of images into different parts like right and left lung. Various handcrafted elements were designed using a trained random forest model. Based on the results of experiments, the sensitivity, specificity, and accuracy are $90.7 \%, 83.3 \%$, and $87.9 \%$. In addition, test results are grouped by the size of the infection, indicating a low sensitivity in patients with minor infections.

\subsection{Disease progression}

Artificial intelligence methods can be used to monitor the progression of the disease, and the progression of the disease can be measured according to the use of different drugs. Medical CT scans are used for this purpose. For example, in a person with COVID-19 disease, the progression of lung infections is significant and vital. By isolating these infections in the lungs (by segmentation algorithms), the progression of the disease can be measured and monitored quickly and automatically. $(40 ; 41 ; 32 ; 30 ; 19)$.

Assessing the disease's progression is very important in medicine, which helps analyze the disease type. For example, in COVID-19 disease, one of the critical components for each patient is to check the level of progression of the virus and infection in the lungs, according to which the patient's treatment should be determined. Unfortunately, the level of disease progression is a difficult task for physicians and requires much time, while the diagnosis and treatment of COVID-19 patients need high-speed methods. This is due to the rapid spread of the disease, and in some cases, the lack of empty beds in hospitals complicates treatment and requires more speed. (42).

Early diagnosis and monitoring of illness progress are greatly aided by computer systems that have been developed in medicine (18). As a result, these technologies have found several medical uses, such as detecting lung cancer and separating a tumor from its surrounding tissue (29; 30). Processing medical data necessitates separating infected lung tissue from the surrounding region $(43 ; 44 ; 45)$. The segmentation of infected tissues can identify the amount of viral dissemination in the lungs, which is critical for patients with COVID-19. For one thing, it is difficult to distinguish diseased lung tissue from surrounding healthy tissue because of this tissue's similarity to its surrounding healthy tissue. As a result, computer systems are unable to distinguish certain sections, such as the lungs, from the rest of the body (46).

Image-based, model-based, and hybrid techniques are examples of segmentation methods. Active Appearance Model (47) and Active Shape Model (32) are examples of model-based techniques. These techniques rely on the image's features (48) to segment it. Only information that appears in the picture is used to segment an image using image-based approaches. Morphological operation; thresholding (19) watershed, level set, active contour, and region growing (20) are some of the image-based approaches that may be used.

Deep learning approaches are commonly utilized to segment ROI in CT scans, which give highquality 3D images for COVID-19 detection and are used to segment ROI in other images as well $(49 ; 50 ; 51)$. Deep networks that are frequently employed for patients with COVID-19 include the traditional U-Net $(52 ; 53 ; 54)$, UNet $++(55)$, and VB-Net $(56 ; 39)$. When compared to CT scanning, X-ray imaging is more widely available across the world. However, dividing X-ray pictures is considerably more difficult since the $2 \mathrm{D}$ projections are projected onto soft textures, which confuses 
the image contrast and makes it difficult to distinguish between them.

COVID-19 application segmentation methodologies may be classified into two types in terms of target ROIs: lung-region-oriented methods and lung-lesion-oriented methods. The lung-regionoriented techniques seek to distinguish lung areas in CT or X-ray, such as the entire lung and lung lobes, from other (background) regions, which is needed in COVID-19 applications. (23; 57; 58). For example, Jin et al. (59) propose a two-step pipeline for screening COVID-19 in CT images, with the first stage consisting of an effective segmentation network based on UNet++ detecting the whole lung region. Lung-lesion-oriented techniques $(60 ; 61)$ aim to distinguish lesions (or metal and motion artifacts) from lung regions. Finding the exact location of a lesion can be difficult because of the variety of sizes, shapes, and textures that lesions can take. It has been suggested that the use of the attention mechanism in screening may be useful in COVID-19 applications in addition to segmentation (62).

There have been multiple ways for lung segmentation in the literature with various aims (63). The U-Net (Fig. 3) is commonly used in COVID applications to segment lung areas and lung lesions. (57; 23). Many U-Net (Fig. ??) and its variations have been created in COVID-19 applications, with acceptable segmentation results. Içek et al. (63) propose a 3D U-Net that uses inter-slice information rather than layers like in a regular U-Net. Military et al. (64) offer the V-Net, which improves the network by using residual blocks as the fundamental convolutional block with a Dice loss. Shan et al. (39) employ a VB-Net for more effective segmentation by providing bottleneck blocks to the convolutional blocks. The UNet++ network suggested by Zhou et al. (65) is substantially more sophisticated than U-Net since it adds a nested convolutional structure between the encodable.

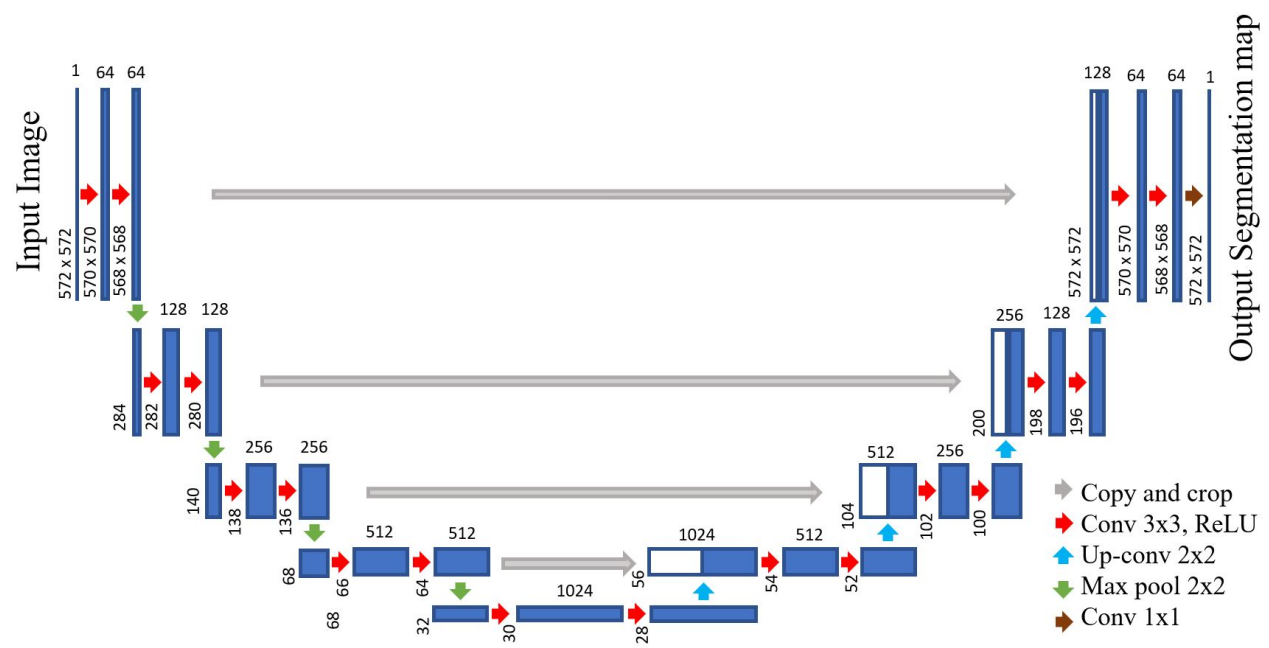

Figure 3: U-Net plan (example for 32x32 pixels in the lowest resolution). Each blue box depicts a multi-channel feature map. The number of channels is stated on the box. TIn the box's lower left border is displayed the $\mathrm{x}-\mathrm{y}$ size. White boxes represent feature map copies. The arrows denote the operations.

Shariaty F. et al. (66) suggested a new ML-based COVID-19 infection segmentation in lung CT images to automatically detect infected areas and their severity. It is vital to address the following 
challenges in deep learning (DL) methods: decrease the computational resource by reducing the dimension of the initial data; and eliminate the critical dependency of the applied classifier on the observed plot. The authors proposed solution to these challenges. They employed statistical parameters derived in blocks of size $\mathrm{m}$ by $\mathrm{m}$ into which the original image is split as input data for the classifier. The author's suggested DL-based segmentation system achieved 0.97 accuracy and 0.97 precision.

AI approaches have a unique position when it comes to modeling COVID-19, which is critical in determining the disorder's future effect. Using these models, policymakers may forecast the future path of the epidemic and plan accordingly. Modeling techniques can take into account the influence of large-scale screening and disease-control measures. ARIMA and LSTM function well in this area, according to the results. Indeed, ARIMA model is the most widely used strategy for predicting time series trends. However, the findings of these research cannot be compared since these approaches have not been used and trained on the same dataset. The pandemic predictions of COVID-19 have been encouraging, however COVID-19 is still an unknown illness with no historical data to forecast its spread, despite the hopeful predictions of artificial intelligence. These methodologies should be integrated into a bigger population of diverse ethnicities in order to develop more accurate predictive models.

COVID-19's stability and growth have been predicted using the ARIMA time technique. The availability of extra datasets has been shown in recent research to improve the model's performance or deliver more precise results. The model's output is based on data gathered from health-care organizations and other sources. As a result, while prediction may not be 100 percent accurate, it may be relied upon as a corrective measure (67). The accuracy of ARIMA can be improved by combining it with new factors and algorithms. Using ARIMA, Adiga et al. (MAPE = 999.1) reported the best performance metrics (68).

\subsection{Individual contact tracing}

Artificial intelligence can assist in analyzing the degree of viral infection by finding clusters and "hot spots," as well as successfully tracking and monitoring people's contacts. In the sense that according to the records of the people and the tests performed, if a person is infected with the virus, by tracking the location of this person, it is possible to identify people suspected of having the disease and places suspected of being infected in the city or region. This can forecast the disease's future course and the chance of recurrence.

If a person has been diagnosed and proven to have COVID-19, the next critical step is to prevent the disease from spreading further. According to the WHO, the virus is spread from person to person primarily by contact with saliva, drops, or nasal discharge (69). Contact tracing is an essential public health method for breaking the virus transmission chain to restrict the spread of SARS-Cov-2. To avoid new outbreaks, the call tracking procedure identifies and manages persons who have recently been exposed to a patient with COVID-19. Generally, this method identifies the afflicted person and provides 14 days of follow-up from the moment of exposure. If this method is appropriately executed, it can break the present novel coronavirus transmission chain, inhibit its spread, and lessen 
the severity of the recent outbreak. In this regard, many infected countries use various technologies such as Bluetooth, Global Positioning System (GPS), social charts, contact details, network-based API, mobile tracking data, card transaction, digital call tracking process with the mobile application. The system's data and physical address. The digital call tracking procedure is quicker and more in real-time than the non-digital system. These digital applications are meant to capture personal data, which is then evaluated by machine learning and artificial intelligence algorithms to follow a person exposed to a new infection due to their recent contact chain.

The Google Scholar publications identify the several countries that have such ML and AL-based call tracking schemes. According to studies, more than 36 countries have effectively implemented digital call tracking in a centralized, decentralized, or a combination of the two approaches to minimize work and boost the efficacy of traditional health care detection systems. (70).

In the case of contact tracking, studies have proven the use of ML and AI in enhancing the contact tracking process against infectious chronic wasting disease (71). After applying graph theory to animal infectious disease epidemic data, mainly inter-farm transport data, the resulting graph properties produced by the proposed model can be used to increase more efficient contact tracking. In addition, the graphs generated have a potential predictive effect on the number of infections that can occur. However, there are still limitations to scenario handling, privacy, data control, and even data security breaches. Countries strive to overcome challenges. Some countries, such as Israel, have enacted an "emergency law for the use of mobile data" to combat the current epidemic (72). Among global call tracking programs, some countries have violated privacy laws and been reported to be insecure (70). So far, they have done a good job by completing the manual tracking process. However, almost every country has its contact tracking plan, as the outbreak continues to spread around the world, becoming a global health emergency. To combat COVID-19 as a unit, we need to provide a proper and standard-focused call tracking application for tracking every human being worldwide. It has also been reported that some specific queries should address this issue: "Is it mandatory or optional?" "Is the effort clear or transparent?" "Has data collection decreased?" "Is the collected information being lost as announced?", "Is the data safe with the host" and "Are there any restrictions or controls on the use of the information?"

Some systems collect the location area corresponding to each phone number from the Call Detailed Record (CDR) provided by the Mobile Network Operators (MNOs) and the medical information (particularly history of the COVID-19 engagement) of each subscriber. In (73), the authors use CDR information to trace, track, and isolate the patients. They adopt information to minimize the widespread coronavirus disease. In (74) and (75), the CDR information is used for criminal investigation. The authors also used the aggregated and anonymized geolocation information from passively collected mobile phone data to inform successfully and model the spatial and temporal dynamics of endemic and emerging infectious diseases, including malaria $(76 ; 77 ; 78 ; 79)$, cholera (80), measles $(81 ; 82)$. 


\section{Conclusions and Discussion}

Since the outbreak of the novel SARS-CoV-2, scientists and medical industries worldwide have been urged to deal with the pandemic by developing alternative methods of rapid screening and prediction, contact tracing, forecasting, and the development of vaccines or drugs that are more accurate and reliable. Machine Learning and Artificial Intelligence are two promising methodologies used by a variety of healthcare providers. This study focuses on recent studies that use such advanced technology to supplement researchers in various ways, addressing the difficulties and obstacles that arise when utilizing such algorithms to support medical experts in real-world issues. This study also contains recommendations conveyed by researchers on AI/ML-based model design, medical specialists, and policymakers on a few faults observed in the current circumstance while dealing with the epidemic. This review demonstrates that using contemporary technology with AI and ML enhances screening, prediction, contact tracking, forecasting, and drug/vaccine development with extraordinary dependability. The majority of the papers used deep learning algorithms, which were shown to be more promising, resilient, and advanced than other learning algorithms. However, given the current urgency, an upgraded model with high-end performance accuracy in screening and predicting SARS-CoV-2 with a distinct type of linked disease by assessing suspects' clinical, mammographic, and demographic information and infected patients is required. Finally, AI and ML may dramatically enhance COVID-19 pandemic management, medicine, screening and prediction, forecasting, contact tracking, and drug/vaccine research while reducing human participation in medical practice. However, most of the models have not been deployed sufficiently to demonstrate their real-world functioning, but they are still capable of fighting the pandemic.

\section{Ethical approval}

All procedures performed in studies involving human participants were by the ethical standards of the institutional and/or national research committee and with the 1964 Helsinki declaration and its later amendments or comparable ethical standards.

\section{References}

[1] E. Dong, H. Du, and L. Gardner, "An interactive web-based dashboard to track covid-19 in real time," The Lancet infectious diseases, vol. 20, no. 5, pp. 533-534, 2020.

[2] T. Singhal, "A review of coronavirus disease-2019 (covid-19)," The indian journal of pediatrics, vol. 87, no. 4, pp. 281-286, 2020.

[3] A. Ozder, "A novel indicator predicts 2019 novel coronavirus infection in subjects with diabetes," diabetes research and clinical practice, vol. 166, p. 108294, 2020.

[4] J. Soltani, I. Sedighi, Z. Shalchi, G. Sami, B. Moradveisi, and S. Nahidi, "Pediatric coronavirus disease 2019 (covid-19): An insight from west of iran," Northern clinics of Istanbul, vol. 7, no. 3, p. 284, 2020. 
[5] A. Sedik, A. M. Iliyasu, A. El-Rahiem, M. E. Abdel Samea, A. Abdel-Raheem, M. Hammad, J. Peng, A. El-Samie, E. Fathi, A. El-Latif et al., "Deploying machine and deep learning models for efficient data-augmented detection of covid-19 infections," Viruses, vol. 12, no. 7, p. 769, 2020.

[6] D. Cyranoski, "'we need to be alert': Scientists fear second coronavirus wave as china's lockdowns ease." Nature, 2020.

[7] I. Mahmud and A. Al-Mohaimeed, "Covid-19: Utilizing local experience to suggest optimal global strategies to prevent and control the pandemic," International Journal of Health Sciences, vol. 14, no. 3, p. 1, 2020.

[8] K. Leung, J. T. Wu, D. Liu, and G. M. Leung, "First-wave covid-19 transmissibility and severity in china outside hubei after control measures, and second-wave scenario planning: a modelling impact assessment," The Lancet, vol. 395, no. 10233, pp. 1382-1393, 2020.

[9] I. Ali, "Covid-19: are we ready for the second wave?" Disaster medicine and public health preparedness, vol. 14, no. 5, pp. e16-e18, 2020.

[10] S. Xu and Y. Li, "Beware of the second wave of covid-19," The Lancet, vol. 395, no. 10233, pp. 1321-1322, 2020.

[11] T. Panch, P. Szolovits, and R. Atun, "Artificial intelligence, machine learning and health systems," Journal of global health, vol. 8, no. 2, 2018.

[12] Z. Hu, Q. Ge, S. Li, L. Jin, and M. Xiong, "Artificial intelligence forecasting of covid-19 in china," arXiv preprint arXiv:2002.07112, 2020.

[13] A. Haleem, M. Javaid, and R. Vaishya, "Effects of covid-19 pandemic in daily life," Current medicine research and practice, vol. 10, no. 2, p. 78, 2020.

[14] H. X. Bai, B. Hsieh, Z. Xiong, K. Halsey, J. W. Choi, T. M. L. Tran, I. Pan, L.-B. Shi, D.-C. Wang, J. Mei et al., "Performance of radiologists in differentiating covid-19 from non-covid-19 viral pneumonia at chest ct," Radiology, vol. 296, no. 2, pp. E46-E54, 2020.

[15] A. Liberati, D. G. Altman, J. Tetzlaff, C. Mulrow, P. C. Gøtzsche, J. P. Ioannidis, M. Clarke, P. J. Devereaux, J. Kleijnen, and D. Moher, "The prisma statement for reporting systematic reviews and meta-analyses of studies that evaluate health care interventions: explanation and elaboration," Journal of clinical epidemiology, vol. 62, no. 10, pp. e1-e34, 2009.

[16] T. Ai, Z. Yang, H. Hou, C. Zhan, C. Chen, W. Lv, Q. Tao, Z. Sun, and L. Xia, "Correlation of chest ct and rt-pcr testing for coronavirus disease 2019 (covid-19) in china: a report of 1014 cases," Radiology, vol. 296, no. 2, pp. E32-E40, 2020.

[17] H. Luo, Q.-L. Tang, Y.-X. Shang, S.-B. Liang, M. Yang, N. Robinson, and J.-P. Liu, "Can chinese medicine be used for prevention of corona virus disease 2019 (covid-19)? a review of historical classics, research evidence and current prevention programs," Chinese journal of integrative medicine, vol. 26, no. 4, pp. 243-250, 2020. 
[18] M. Ebrahimi, B. Novikov, E. Ebrahimie, A. Spilman, R. Ahsan, M. R. Tahsili, M. Najafi, S. Navvabi, and F. Shariaty, "The first report of the most important sequential differences between covid-19 and mers viruses by attribute weighting models, the importance of nucleocapsid (n) protein," 2020.

[19] F. Shariaty, S. Hosseinlou, and V. Y. Rud, "Automatic lung segmentation method in computed tomography scans," in Journal of Physics: Conference Series, vol. 1236, no. 1. IOP Publishing, 2019, p. 012028.

[20] M. Mousavi, F. Shariaty, M. Orooji, and E. Velichko, "The performance of active-contour and region growing methods against noises in the segmentation of computed-tomography scans," in International Youth Conference on Electronics, Telecommunications and Information Technologies. Springer, 2021, pp. 573-582.

[21] F. Pan, T. Ye, P. Sun, S. Gui, B. Liang, L. Li, D. Zheng, J. Wang, R. L. Hesketh, L. Yang et al., "Time course of lung changes on chest ct during recovery from 2019 novel coronavirus (covid-19) pneumonia," Radiology, 2020.

[22] J. Chen, L. Wu, J. Zhang, L. Zhang, D. Gong, Y. Zhao, Q. Chen, S. Huang, M. Yang, X. Yang et al., "Deep learning-based model for detecting 2019 novel coronavirus pneumonia on highresolution computed tomography," Scientific reports, vol. 10, no. 1, pp. 1-11, 2020.

[23] C. Zheng, X. Deng, Q. Fu, Q. Zhou, J. Feng, H. Ma, W. Liu, and X. Wang, "Deep learning-based detection for covid-19 from chest ct using weak label," MedRxiv, 2020.

[24] C. Jin, W. Chen, Y. Cao, Z. Xu, X. Zhang, L. Deng, C. Zheng, J. Zhou, H. Shi, and J. Feng, "Development and evaluation of an ai system for covid-19 diagnosis. medrxiv 2020," preprint [https://doi. org/10.1101/2020.03. 20.20039834].

[25] H. Mukherjee, S. Ghosh, A. Dhar, S. M. Obaidullah, K. Santosh, and K. Roy, "Deep neural network to detect covid-19: one architecture for both ct scans and chest x-rays," Applied Intelligence, vol. 51, no. 5, pp. 2777-2789, 2021.

[26] S. H. Kassania, P. H. Kassanib, M. J. Wesolowskic, K. A. Schneidera, and R. Detersa, "Automatic detection of coronavirus disease (covid-19) in x-ray and ct images: a machine learning based approach," Biocybernetics and Biomedical Engineering, vol. 41, no. 3, pp. 867-879, 2021.

[27] A. M. Luks and E. R. Swenson, "Covid-19 lung injury and high-altitude pulmonary edema. a false equation with dangerous implications," Annals of the American Thoracic Society, vol. 17, no. 8, pp. 918-921, 2020.

[28] A. UR and K. Verma, "Pulmonary edema in covid19 - a neural hypothesis," ACS Chemical Neuroscience, vol. 11, no. 14, pp. 2048-2050, 2020.

[29] F. Shariaty and M. Mousavi, "Application of cad systems for the automatic detection of lung nodules," Informatics in Medicine Unlocked, vol. 15, p. 100173, 2019. 
[30] F. Shariaty, V. Davydov, V. Yushkova, A. Glinushkin, and V. Y. Rud, "Automated pulmonary nodule detection system in computed tomography images based on active-contour and svm classification algorithm," in Journal of Physics: Conference Series, vol. 1410, no. 1. IOP Publishing, 2019, p. 012075.

[31] W.-j. Guan, Z.-y. Ni, Y. Hu, W.-h. Liang, C.-q. Ou, J.-x. He, L. Liu, H. Shan, C.-l. Lei, D. S. Hui et al., "Clinical characteristics of coronavirus disease 2019 in china," New England journal of medicine, vol. 382, no. 18, pp. 1708-1720, 2020.

[32] F. Shariaty, M. Orooji, M. Mousavi, M. Baranov, and E. Velichko, "Automatic lung segmentation in computed tomography images using active shape model," in 2020 IEEE International Conference on Electrical Engineering and Photonics (EExPolytech). IEEE, 2020, pp. 156-159.

[33] A. Bernheim, X. Mei, M. Huang, Y. Yang, Z. A. Fayad, N. Zhang, K. Diao, B. Lin, X. Zhu, $\mathrm{K}$. Li et al., "Chest ct findings in coronavirus disease-19 (covid-19): relationship to duration of infection," Radiology, p. 200463, 2020.

[34] H. Chiroma, A. E. Ezugwu, F. Jauro, M. A. Al-Garadi, I. N. Abdullahi, and L. Shuib, "Early survey with bibliometric analysis on machine learning approaches in controlling covid-19 outbreaks," PeerJ Computer Science, vol. 6, p. e313, 2020.

[35] S. Wang, B. Kang, J. Ma, X. Zeng, M. Xiao, J. Guo, M. Cai, J. Yang, Y. Li, X. Meng et al., "A deep learning algorithm using ct images to screen for corona virus disease (covid-19)," MedRxiv, 2020 .

[36] Y. Song, S. Zheng, L. Li, X. Zhang, X. Zhang, Z. Huang, J. Chen, H. Zhao, Y. Jie, R. Wang et al., "Deep learning enables accurate diagnosis of novel coronavirus (covid-19) with ct images," MedRxiv, 2020.

[37] X. Xu, X. Jiang, C. Ma, P. Du, X. Li, S. Lv, L. Yu, Q. Ni, Y. Chen, J. Su et al., "A deep learning system to screen novel coronavirus disease 2019 pneumonia," Engineering, vol. 6, no. 10, pp. 1122-1129, 2020.

[38] F. Shi, L. Xia, F. Shan, D. Wu, Y. Wei, H. Yuan, H. Jiang, Y. Gao, H. Sui, and D. Shen, "Large-scale screening of covid-19 from community acquired pneumonia using infection sizeaware classification," arXiv preprint arXiv:2003.09860, 2020.

[39] F. Shan, Y. Gao, J. Wang, W. Shi, N. Shi, M. Han, Z. Xue, and Y. Shi, "Lung infection quantification of covid-19 in ct images with deep learning," arXiv preprint arXiv:2003.04655, 2020 .

[40] J. Stebbing, A. Phelan, I. Griffin, C. Tucker, O. Oechsle, D. Smith, and P. Richardson, "Covid19: combining antiviral and anti-inflammatory treatments," The Lancet Infectious Diseases, vol. 20, no. 4, pp. 400-402, 2020. 
[41] A. Haleem, R. Vaishya, M. Javaid, and I. H. Khan, "Artificial intelligence (ai) applications in orthopaedics: an innovative technology to embrace," Journal of clinical orthopaedics and trauma, vol. 11, no. Suppl 1, p. S80, 2020.

[42] E. E.-D. Hemdan, M. A. Shouman, and M. E. Karar, "Covidx-net: A framework of deep learning classifiers to diagnose covid-19 in x-ray images," arXiv preprint arXiv:2003.11055, 2020.

[43] D.-P. Fan, T. Zhou, G.-P. Ji, Y. Zhou, G. Chen, H. Fu, J. Shen, and L. Shao, "Inf-net: Automatic covid-19 lung infection segmentation from ct images," IEEE Transactions on Medical Imaging, vol. 39, no. 8, pp. 2626-2637, 2020.

[44] S. Hassantabar, M. Ahmadi, and A. Sharifi, "Diagnosis and detection of infected tissue of covid19 patients based on lung x-ray image using convolutional neural network approaches," Chaos, Solitons $\& 5$ Fractals, vol. 140, p. 110170, 2020.

[45] V. Pavlov, F. Shariaty, M. Orooji, and E. Velichko, "Application of deep learning techniques for detection of covid-19 using lung ct scans: Model development and validation."

[46] M. Baranov, E. Velichko, and A. Andryakov, "Image processing for analysis of bio-liquid films," Optical Memory and Neural Networks, vol. 29, pp. 1-6, 2020.

[47] F. Shariaty, M. Orooji, N. E. Velichko, and V. S. Zavjalov, "Texture appearance model, a new model-based segmentation paradigm, application on the segmentation of lung nodule in the ct scan of the chest," Computers in Biology and Medicine, p. 105086, 2021.

[48] F. Shariaty, M. Baranov, E. Velichko, M. Galeeva, and V. Pavlov, "Radiomics: extracting more features using endoscopic imaging," in 2019 IEEE International Conference on Electrical Engineering and Photonics (EExPolytech). IEEE, 2019, pp. 181-194.

[49] X. Chen, L. Yao, and Y. Zhang, "Residual attention u-net for automated multi-class segmentation of covid-19 chest ct images," arXiv preprint arXiv:2004.05645, 2020.

[50] L. Zhou, Z. Li, J. Zhou, H. Li, Y. Chen, Y. Huang, D. Xie, L. Zhao, M. Fan, S. Hashmi et al., "A rapid, accurate and machine-agnostic segmentation and quantification method for ct-based covid-19 diagnosis," IEEE transactions on medical imaging, vol. 39, no. 8, pp. 2638-2652, 2020.

[51] F. Shi, J. Wang, J. Shi, Z. Wu, Q. Wang, Z. Tang, K. He, Y. Shi, and D. Shen, "Review of artificial intelligence techniques in imaging data acquisition, segmentation and diagnosis for covid-19," IEEE reviews in biomedical engineering, 2020.

[52] N. Saeedizadeh, S. Minaee, R. Kafieh, S. Yazdani, and M. Sonka, "Covid tv-unet: Segmenting covid-19 chest ct images using connectivity imposed u-net," arXiv preprint arXiv:200\%.12303, 2020 .

[53] D. Müller, I. S. Rey, and F. Kramer, "Automated chest ct image segmentation of covid-19 lung infection based on 3d u-net," arXiv preprint arXiv:2007.04774, 2020. 
[54] T. Zhou, S. Canu, and S. Ruan, "An automatic covid-19 ct segmentation based on u-net with attention mechanism," arXiv preprint arXiv:2004.06673, 2020.

[55] Y. Lu, X. Qin, H. Fan, T. Lai, and Z. Li, "Wbc-net: A white blood cell segmentation network based on unet++ and resnet," Applied Soft Computing, vol. 101, p. 107006, 2021.

[56] Y. Wang, Y. Chen, Y. Wei, M. Li, Y. Zhang, N. Zhang, S. Zhao, H. Zeng, W. Deng, Z. Huang et al., "Quantitative analysis of chest ct imaging findings with the risk of ards in covid-19 patients: a preliminary study," Annals of translational medicine, vol. 8, no. 9, 2020.

[57] Y. Cao, Z. Xu, J. Feng, C. Jin, X. Han, H. Wu, and H. Shi, "Longitudinal assessment of covid19 using a deep learning-based quantitative ct pipeline: Illustration of two cases," Radiology: Cardiothoracic Imaging, vol. 2, no. 2, p. e200082, 2020.

[58] L. Huang, R. Han, T. Ai, P. Yu, H. Kang, Q. Tao, and L. Xia, "Serial quantitative chest ct assessment of covid-19: a deep learning approach," Radiology: Cardiothoracic Imaging, vol. 2, no. 2 , p. e200075, 2020.

[59] S. Jin, B. Wang, H. Xu, C. Luo, L. Wei, W. Zhao, X. Hou, W. Ma, Z. Xu, Z. Zheng et al., "Ai-assisted ct imaging analysis for covid-19 screening: Building and deploying a medical ai system in four weeks," MedRxiv, 2020.

[60] L. Tang, X. Zhang, Y. Wang, and X. Zeng, "Severe covid-19 pneumonia: assessing inflammation burden with volume-rendered chest ct," Radiology: Cardiothoracic Imaging, vol. 2, no. 2, p. e200044, 2020.

[61] C. Shen, N. Yu, S. Cai, J. Zhou, J. Sheng, K. Liu, H. Zhou, Y. Guo, and G. Niu, "Quantitative computed tomography analysis for stratifying the severity of coronavirus disease 2019," Journal of pharmaceutical analysis, vol. 10, no. 2, pp. 123-129, 2020.

[62] G. Gaál, B. Maga, and A. Lukács, "Attention u-net based adversarial architectures for chest x-ray lung segmentation," arXiv preprint arXiv:2003.10304, 2020.

[63] Ö. Çiçek, A. Abdulkadir, S. S. Lienkamp, T. Brox, and O. Ronneberger, "3d u-net: learning dense volumetric segmentation from sparse annotation," in International conference on medical image computing and computer-assisted intervention. Springer, 2016, pp. 424-432.

[64] F. Milletari, N. Navab, and S.-A. Ahmadi, "V-net: Fully convolutional neural networks for volumetric medical image segmentation," in 2016 fourth international conference on $3 D$ vision $(3 D V)$. IEEE, 2016, pp. 565-571.

[65] Z. Zhou, M. M. R. Siddiquee, N. Tajbakhsh, and J. Liang, "Unet++: A nested u-net architecture for medical image segmentation," in Deep learning in medical image analysis and multimodal learning for clinical decision support. Springer, 2018, pp. 3-11.

[66] F. Shariaty, V. Pavlov, E. Velichko, T. Pervunina, and M. Orooji, "Severity and progression quantification of covid-19 in ct images: a new deep-learning approach," in 2021 International Conference on Electrical Engineering and Photonics (EExPolytech). IEEE, 2021, pp. 72-76. 
[67] D. Painuli, D. Mishra, S. Bhardwaj, and M. Aggarwal, "Forecast and prediction of covid-19 using machine learning," in Data Science for COVID-19. Elsevier, 2021, pp. 381-397.

[68] A. Adiga, L. Wang, B. Hurt, A. S. Peddireddy, P. Porebski, S. Venkatramanan, B. Lewis, and M. Marathe, "All models are useful: Bayesian ensembling for robust high resolution covid-19 forecasting," medRxiv, 2021.

[69] W. H. Organization et al., "Coronavirus disease 2019 (covid-19): situation report, 73," 2020.

[70] P. O'Neill, T. Ryan-Mosley, and B. Johnson, "A flood of coronavirus apps are tracking us," Now it's time to keep track of them, 2020.

[71] C. Rorres, M. Romano, J. A. Miller, J. M. Mossey, T. H. Grubesic, D. E. Zellner, and G. Smith, "Contact tracing for the control of infectious disease epidemics: Chronic wasting disease in deer farms," Epidemics, vol. 23, pp. 71-75, 2018.

[72] J. Tidy, "Coronavirus: Israel enables emergency spy powers," BBC News, vol. 17, 2020.

[73] S. Nisar, M. A. Zuhaib, A. Ulasyar, and M. Tariq, "A privacy-preserved and cost-efficient control scheme for coronavirus outbreak using call data record and contact tracing," IEEE Consumer Electronics Magazine, vol. 10, no. 2, pp. 104-110, 2020.

[74] S. Khan, F. Ansari, H. A. Dhalvelkar, and S. Computer, "Criminal investigation using call data records (cdr) through big data technology," in 2017 International Conference on Nascent Technologies in Engineering (ICNTE). IEEE, 2017, pp. 1-5.

[75] M. Kumar, M. Hanumanthappa, and T. S. Kumar, "Crime investigation and criminal network analysis using archive call detail records," in 2016 Eighth International Conference on Advanced Computing (ICoAC). IEEE, 2017, pp. 46-50.

[76] A. Wesolowski, N. Eagle, A. J. Tatem, D. L. Smith, A. M. Noor, R. W. Snow, and C. O. Buckee, "Quantifying the impact of human mobility on malaria," Science, vol. 338, no. 6104, pp. 267-270, 2012.

[77] N. W. Ruktanonchai, P. DeLeenheer, A. J. Tatem, V. A. Alegana, T. T. Caughlin, E. zu ErbachSchoenberg, C. Lourenço, C. W. Ruktanonchai, and D. L. Smith, "Identifying malaria transmission foci for elimination using human mobility data," PLoS computational biology, vol. 12, no. 4, p. e1004846, 2016.

[78] D. K. Pindolia, A. J. Garcia, A. Wesolowski, D. L. Smith, C. O. Buckee, A. M. Noor, R. W. Snow, and A. J. Tatem, "Human movement data for malaria control and elimination strategic planning," Malaria journal, vol. 11, no. 1, pp. 1-16, 2012.

[79] A. J. Tatem, Z. Huang, C. Narib, U. Kumar, D. Kandula, D. K. Pindolia, D. L. Smith, J. M. Cohen, B. Graupe, P. Uusiku et al., "Integrating rapid risk mapping and mobile phone call record data for strategic malaria elimination planning," Malaria journal, vol. 13, no. 1, pp. $1-16,2014$. 
[80] C. M. Peak, A. L. Reilly, A. S. Azman, and C. O. Buckee, "Prolonging herd immunity to cholera via vaccination: Accounting for human mobility and waning vaccine effects," PLoS neglected tropical diseases, vol. 12, no. 2, p. e0006257, 2018.

[81] Y. Xia, O. N. Bjørnstad, and B. T. Grenfell, "Measles metapopulation dynamics: a gravity model for epidemiological coupling and dynamics," The American Naturalist, vol. 164, no. 2, pp. 267-281, 2004.

[82] B. T. Grenfell, O. N. Bjørnstad, and J. Kappey, "Travelling waves and spatial hierarchies in measles epidemics," Nature, vol. 414, no. 6865, pp. 716-723, 2001. 